\title{
Inducción del parto versus manejo expectante en ruptura prematura de membranas pretérmino: revisión sistemática y metanálisis
}

\author{
Induction of labor versus expectant management in \\ patients with preterm premature rupture of membranes: \\ a systematic review and meta-analysis
}

\author{
Diego A Téllez G ${ }^{1}$, Santiago Ramírez $\mathrm{F}^{1}$, Nathalia S Parada B ${ }^{1}$, Julián A Fernández-Niño ${ }^{1}$
}

Forma de citar: Téllez DA, Ramírez S, Parada NS, Fernández-Niño JA. Inducción del parto versus menejo expectante en ruptura prematura de membranas pretérmino: revisión sistemática y metanálisis. Rev Univ Ind Santander Salud. 2017; 49(1): 45-55. DOI: http://dx.doi.org/10.18273/revsal.v49n1-2017005 @) (1)

\section{RESUMEN}

Introducción: La ruptura prematura de membranas pretérmino (PPROM) es una patología obstétrica que genera un alto riesgo de morbilidad fetal. Pese a esto, actualmente prevalece la incertidumbre ante el manejo adecuado para ésta entidad. Objetivo: Comparar los desenlaces materno-fetales de la inducción del parto (IL) y manejo expectante (EM) Metodología: Revisión sistemática y metanálisis Fuentes de datos: Se buscó en las bases de datos MEDLINE, EMBASE, SCIELO y Google Académico. Criterios de elección, pacientes e intervenciones: Se incluyeron ensayos clínicos aleatorizados controlados y estudios de cohortes que compararan el EM e IL en pacientes con PPROM entre la semana 24 a 36 6/7, y que adicionalmente tuvieran en cuenta como desenlaces analizados: sepsis neonatal (NS), síndrome de distrés respiratorio (SDR), muerte neonatal y corioamionitis. Recolección de datos y análisis: La extracción y registro de datos se ejecutó por dos revisores de forma independiente. La evaluación de calidad se realizó con CONSORT y STROBE, según corresponda. Metanálisis: Para cada desenlance, se realizó la estimación del Riesgo Relativo (RR) consolidado, usando modelos de efectos aleatorios. Resultados: Un total de 3378 mujeres con PPROM fueron incluidas en los 9 estudios (8 ensayos clínicos). En el meta-análisis no se encontraron diferencias significativas en la ocurrencia de SDR ( $R R=1,08$; IC 95\%: 0,89-1,31), ni de sepsis neonatal ( $R R=0,92$; IC 95\%: 0,61-1,39), en los tratados con IL comparados con el EM. Tampoco se encontraron diferencias significativas en la ocurrencia de muertes neonatales ( $R R=1,34$; IC 95\%: 0,93-1,93) ni corioamnionitis $(R R=0,88$; IC 95\%: 0,581,35). Conclusiones: Los resultados sugieren que no existe evidencia de diferencias estadísticamente significativas en la ocurrencia de los principales desenlaces cuando se compara EM con IL. Más estudios y con mayores tamaños de muestras son necesarios.

Palabras clave: Ruptura prematura de membranas prétermino, Inducción del parto, Síndrome de distrés respiratorio neonatal, Corioamnionitis, Manejo expectante.

1. Universidad Industrial de Santander. Bucaramanga, Colombia

Correspondencia: Nathalia Stefanía Parada Botello. Estudiante de medicina. Cll 32 28-51 Edificio Astoria Plaza. Bucaramanga, Colombia. Tel: (+57)7 6900206. Correo electrónico: nparadab@hotmail.com. 


\section{ABSTRACT}

Introduction: Preterm premature rupture of membranes (PPROM), is an obstetric pathology that causes a high-risk of morbidities and higher rate of hospital readmission in the first month of life. However, the management of this patology is still uncertain. Objetive: To compare maternal-fetal outcomes of induction of labor (IL) and expectant management (EM) in order to determine the actions to follow. Methodology: Systematic review and meta-analysis Data collection: We searched MEDLINE, EMBASE, SCIELO and Google Scholar. Selection Criteria, patients and interventions: Controlled randomized clinical trial and cohort studies were included. These studies compared the EM and IL in patients with PPROM within 24 to 36 6/7 weeks and take into account outcomes such as neonatal sepsis (NS), respiratory distress syndrome (RDS), neonatal death or chorioamnionitis. Analysis and data collection: Two authors independently executed the extraction and recording of data. Quality assessment was performed with the CONSORT or STROBE score, accordingly. Meta-analysis: For each outcome, a pooled Relative Risk was estimated using random effects models. Results 3378 women with PPROM were included in 9 studies (8 clinical trials). In the meta-analysis, we did not find a statistically significant differences in the occurrence of RDS (RR = 1.08; 95\% CI: 0.89-1.31 or NS (RR=0.92; IC 95\%: 0.61-1.39), in the IL group in comparison with EM. We did not find either differences in the occurrence of neonatal deaths ( $R R=1.34$; IC 95\%: 0.93-1.93) or chorioamnionitis ( $\mathrm{RR}=0.88$; IC 95\%: 0.58-1.35). Conclusions: The results suggest that there is no evidence of statistically significant differences in the occurrence of major outcomes when comparing MS with IL. Further studies and larger sample sizes will be necesary.

Keywords: Preterm Premature Rupture of Membranes, Induction of labor, Watchful Waitings, Neonatal Respiratory Distress Syndrome, Chorioamnionitis.

\section{INTRODUCCIÓN}

La ruptura prematura de membranas pretérmino (PPROM) se define como la ruptura de las membranas previa al inicio de actividad uterina antes de la semana 37 de gestación ${ }^{1}$. Este suceso presenta una incidencia a nivel mundial cercana al $3 \%$, y se encuentra asociado con estatus socioeconómico bajo $^{2}$, antecedente de parto pretérmino, infecciones de vías urinarias y hemorragias durante el embarazo ${ }^{3}$. La PPROM es considerada actualmente como el principal agente causal del 30\% de los partos pretérmino ${ }^{4}$, representando un alto riesgo de morbilidad fetal y una alta tasa de hospitalización asociada en el primer mes de vida comparados con los recién nacidos a término ${ }^{5}$. Asimismo, genera un aumento en la posibilidad de producir efectos adversos en el desarrollo fetal secundario a un estado de oligoamnios prolongado ${ }^{6}$.

En Colombia, durante el año 2014, según estadísticas del Departamento Administrativo Nacional de Estadística (DANE), el 19\% de los partos fueron pretérmino de los cuales el $9 \%$ se asoció a mortalidad fetal ${ }^{7}$. Estas estadísticas han venido en aumento comparadas con las presentadas en el año 2004, donde la incidencia de parto pretérmino fue del $16,1 \%{ }^{3}$, siendo la mayoría de estos partos producto de PPROM.

Por lo que refiere al manejo óptimo de los embarazos complicados con PPROM, a pesar de la existencia de revisiones sistemáticas y metanálisis previos, en la actualidad, este sigue siendo un asunto de gran controversia en la obstetricia ${ }^{8}$ pues no existe un consenso que indique con certeza el modo exacto de proceder ante esta situación para generar el mejor desenlace para el binomio madre-neonato. Entre las opciones que actualmente se emplean al inicio de la presentación del cuadro se encuentran el uso de tocolíticos, la administración de antibiótico de profilaxis y esquema de maduración pulmonar; además del manejo expectante (EM) versus la inducción del trabajo de parto (IL) ${ }^{9}$, siendo estos dos últimos los más debatibles en cuestión dado que se encuentran directamente ligados con la edad gestacional ${ }^{10}$ al momento del parto; y por lo tanto las complicaciones que esto acarrea.

Es por todo lo anterior, que consideramos de gran importancia continuar con la búsqueda, evaluación y síntesis de literatura más actualizada para establecer si existen diferencias estadísticamente significativas en los desenlaces de sepsis neonatal (NS), síndrome de distrés respiratorio (SDR), muerte neonatal y corioamnionitis, en las gestantes tratadas con IL comparadas con las manejadas con EM, con el fin de determinar basados en la mejor evidencia disponible, cuál sería la mejor conducta terapeútica a seguir con mayores resultados favorables, y menores riesgos, tanto para la madre y el neonato. 


\section{METODOLOGÍA}

PICO y Estrategia de búsqueda: Se realizó una búsqueda sistemática de la literatura tomando como guía la realización de la pregunta PICO, con el fin de identificar publicaciones científicas que incluyeran nuestra población diana, esta es: pacientes con ruptura prematura de membranas pretérmino; en donde se comparan las dos alternativas de manejo mencionadas: Inducción del trabajo de parto (IL) y manejo expectante (EM); y que considerarán al menos uno de los siguientes cuatro desenlaces: sepsis neonatal, síndrome de distrés respiratorio, muerte neonatal y corioamnionitis.

La búsqueda se realizó en las bases de datos MEDLINE, EMBASE y SCIELO hasta septiembre del 2016, combinando 8 palabras claves utilizando operadores booleanos que representaban en su totalidad el problema planteado en la PICO: "preterm premature rupture of membranes", "neonatal outcome", "microbial invation of the amniotic cavity", "neonatal respiratory distress syndrome", "fetal outcome", "watchful waitings" e "induction of labor". No se determinó un límite para la antigüedad de los estudios, ni se hizo ninguna restricción de lenguaje. Para tratar de abarcar la evidencia no publicada existente (literatura gris), se realizó además una búsqueda en Google Académico, como también se escribió a algunos autores más representativos sobre el tema consultando sobre la existencia de otros trabajos no publicados, y se hizo una búsqueda en bola de nieve en otras revisiones, usando listas de referencias de los artículos elegidos.

El proceso de selección de artículos se produjo de manera pareada e independiente, en primera instancia por título - desde la base de datos, dos miembros del equipo escogían artículos según su criterio y un tercero revisaba esa inclusión valorando otra vez dicha selección - y posteriormente por "abstract", a través del mismo sistema.

Criterios, y procedimientos de selección de los estudios: Se escogieron publicaciones que compararan la inducción del parto y el manejo expectante en pacientes con ruptura prematura de membranas pretérmino; que asimismo cumplieran las siguientes condiciones: (I) La PPROM ocurriera entre la semana 24 a 36 6/7 en las pacientes incluidas; (II) Inclusión del análisis de al menos una de las cuatro complicaciones mencionadas previamente (SDR, NS, muerte neonatal y corioamnionitis); (III) Estudios de tipo ensayo clínico aleatorizado controlado (ECAC) o estudios de cohortes; (IV) Presencia de manejo expectante e inducción de labor de parto como grupos de comparación.
Se excluyeron estudios donde la muestra de estudio presentara comorbilidades previas $\mathrm{u}$ otras complicaciones asociadas al embarazo distintas a la ruptura prematura de membrana pretérmino (trastornos hipertensivos, diabetes gestacional, trastornos hepáticos, infección materna y perinatal $)(n=2)$ y aquellas investigaciones que sólo incluyeran exclusivamente pacientes con embarazo múltiple $(\mathrm{n}=2)$.

Extracción de datos y evaluación de calidad: De cada artículo se extrajo la siguiente información: autores, año de publicación, país, tipo de estudio, periodo de estudio, número de intervenidos y eventos en cada grupo; y los resultados medidos, haciendo énfasis en sepsis neonatal y síndrome de distrés respiratorio como desenlaces primarios y, corioamnionitis y mortalidad neonatal como secundarios. La extracción y registro de datos se ejecutó por dos revisores de forma independiente. Cada uno de ellos fue cegado a los nombres de revistas, instituciones o donaciones de fondos. Cualquier discrepancia entre estos dos revisores se resolvió mediante un tercer revisor, que también extrajo los datos y participó en la discusión con ellos y fue encargado de la decisión final. Finalmente, para los ensayos clínicos se evalúo la calidad de cada estudio, aplicando la escala CONSORT, y para los estudios de cohortes la escala STROBE. Esta evaluación también fue realizada de madera pareada, y se presenta el promedio obtenido de ambos evaluador. Dada la poca evidencia disponible, no se realizaron exclusiones con el criterio de calidad; aunque todos los estudios tuvieron un nivel moderado o alto.

Análisis estadístico: El metanálisis se realizó para cada uno de los cuatro desenlaces de interés: síndrome de distrés respiratorio, sepsis neonatal, corioamninotis y muerte neonatal. En primer lugar, a partir de la información primaria de los estudios, se reconstruyeron las tablas de contingencia y se estimaron los Riesgos Relativos (RR) con su respectivo intervalo de confianza. La mayoría de los estudios no reportaban ningún estimador de asociación, así que estos debieron ser construidos por los autores, a partir de los datos originales de cada estudio. Para cada desenlace, se aplicaron pruebas tipo ji-cuadrada de heterogeneidad y la prueba Tau-cuadrada y se estimó la I2. Debido a que se encontró una heterogeneidad moderada (50 -75\%) para todos los desenlaces, y dado que las pruebas de heterogeneidad fueron estadísticamente significativas a un alfa de 0,05 , se decidió ajustar modelos de efectos aleatorios para estimar los RR consolidados.

Los RR consolidados fueron estimados para cada desenlace y son presentados en Forest- Plot. Los estimadores obtenidos en cada metanálisis fueron 
comparados, con y sin incluir el estudio de cohorte, en un análisis de sensibilidad, y no se encontraron diferencias sistemáticas en los resultados, lo cuál hizo razonable su inclusión, especialmente dada su alta calidad, y que no afecta el estimador obtenido. Una meta-regresión para cada desenlace fue realizada, usando una variable indicadora de la edad gestacional al momento del PPROM como variable independiente principal, donde " 1 " era 28 a 34 semanas, y " 0 " mayor de 34 semanas; esta categorización fue realizada así por corresponder a los rangos reportados en los estudios. Otras potenciales fuentes de heterogenidad no pudieron ser analizadas al no estar disponible la información en la mayoría de estudios. Finalmente, se realizó el test de Egger para explorar el potencial sesgo de publicación. Todos los análisis fueron realizados en STATA 14 (Stata Corporation, College Station, TX, USA).

\section{RESULTADOS}

Estudios obtenidos y seleccionados: En la búsqueda se incluyeron las palabras (MESH) mencionadas, combinadas con operadores booleanos, en MEDLINE, EMBASE, SCIELO y Google Académico, obteniendo inicialmente un total de 524 artículos. En un primer momento, se realizó la selección pareada por títulos, donde fueron seleccionados 72 artículos, de los cuales sólo 15 estudios cumplieron con los criterios de inclusión especificados en la sección de métodos. Posteriormente, cuatro estudios fueron eliminados por tratarse de presentaciones en congreso, y dado que no se logró encontrar el trabajo final de ninguno de ellos. Finalmente dos estudios fueron excluidos, uno por presentar población con comorbilidades de base y un segundo estudio por no poseer los grupos de tratamiento requeridos para esta revisión. En total, se obtuvieron al final nueve estudios, de los cuáles ocho fueron ensayos clínicos. Los artículos seleccionados se publicaron entre 1981 y 2015. El flujograma del procedimiento de selección es presentado en la FIGURA 1.

Descripción general de los diseños encontrados y análisis de calidad: La TABLA 1 muestra las características generales de cada uno de los estudios seleccionados. Aleatorización: Los estudios incluidos en la revisión realizaron aleatorización en su mayoría (7/9), excepto los realizados por Eroiz-Hernandez J, et al. ${ }^{11}$; y Pasquier J, et al. ${ }^{12}$, este último obviamente por tratarse de un estudio de cohortes. A pesar de que no hay grandes diferencias entre el número de pacientes en los dos grupos de intervención de cada estudio,
Van der Ham DP, et al. ${ }^{13}$, Morris J, et al. ${ }^{14}$ y un segundo estudio de Van der Ham DP, et al. ${ }^{15}$ especifican que la aleatorización se realizó 1:1 mediante una base de datos computarizada, este mismo sistema fue usado por Mercer $\mathrm{BM}$, et $\mathrm{al}^{16}$, pero no indicaron en qué proporción se realizó la aleatorización, o la estrategía utilizada; por su parte Cox S, et al. ${ }^{17}$ y Iams JD, et al. ${ }^{18}$ especifican que realizaron la aleatorización mediante asignación al azar por medio de una tabla de números aleatorios. Ciegos: La aplicación de ciegos en los estudios sólo se realizó al momento de la aleatorización en siete de los nueve estudios, pero los pacientes y los cuidadores en todos los estudios si conocían al final a que grupo de tratamiento fueron asignados. Pasquier J, et al. ${ }^{12}$ y Iams JD, et al. ${ }^{18}$ no mencionan la realización de ningún tipo de ciegos. Seguimiento: El seguimiento de las pacientes se realizó dentro del ámbito hospitalario por lo cual en la mayoría de los estudios no se presentaron perdidas en el seguimiento. Únicamente los estudios realizados por Morris J, et al. ${ }^{14}$ y Iams JD, et al. ${ }^{18}$ presentaron dos y tres pérdidas en el seguimiento respectivamente, en el caso de Iams JD, et al ${ }^{18}$ la pérdida se presentó en pacientes asignadas en el grupo de EM donde las pacientes solo eran admitidas en el centro hospitalario antes del parto. Evaluación de calidad: Los resultados de la evaluación de la calidad del estudio pueden ser solicitados a los autores. De los nueve artículos, siete son de calidad alta y de dos de calidad moderada.

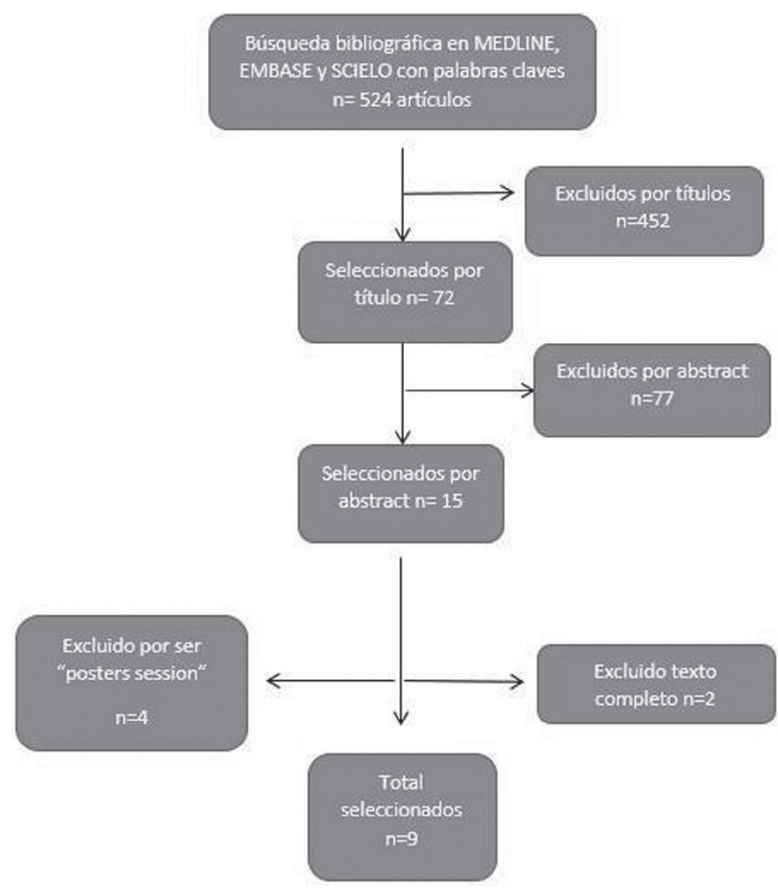

Figura 1. Flujograma de búsqueda. 
Características de la muestra estudiada: Un total de 3378 mujeres con PPROM fueron incluidas en los nueve estudios, de las cuales 1672 fueron tratadas con IL y 1706 con EM. La Tabla 1 presenta también las características de la muestra en cada estudio. En la totalidad de las publicaciones incluidas se presentaron mujeres embarazadas complicadas con PPROM de diferentes edades gestacionales. Van der Ham DP, et al. ${ }^{13}$, Morris J, et al. ${ }^{14} \mathrm{y}$ un segundo estudio de Van der Ham DP, et al. ${ }^{15}$ incluyeron pacientes con PPROM entre las semanas 34 y $366 / 7$, mientras que los pacientes incluidas en los estudios Iams JD, et al. ${ }^{18}$, Garite TJ, et al. ${ }^{19}$ y Eroiz-
Hernández J, et al. ${ }^{11}$ sufrieron PPROM entre la semana 28-34. Cox S, et al. ${ }^{17}$ y Mercer BM, et al. ${ }^{16}$ incluyeron pacientes con PPROM en las semanas 30-34 y 32-36 respecivamente. Por otro lado, Pasquier J, et al. ${ }^{12}$ fueron los únicos que incluyeron pacientes con PPROM desde la semana 24 hasta la $336 / 7$. Cox S, et al ${ }^{17}$ y un estudio de Van der Ham DP, et a $1^{15}$ incluyeron dos y seis pacientes con embarazo gemelar, respectivamente. La edad gestacional en las pacientes incluidas en todos los estudios fue determinada por la fecha de última menstruación o por la ecografía más temprana, proporcionando de esta manera una mayor precisión.

TABLA 1. Características generales de los estudios incluidos en la revisión

\begin{tabular}{|c|c|c|c|c|c|}
\hline Primer autor; año & País & Tipo de estudio & Periodo de estudio & $\begin{array}{c}\text { Rango de edad } \\
\text { gestacional a } \\
\text { PPROM }^{\mathrm{a}}\end{array}$ & $\begin{array}{c}\text { Muestra } \\
\left(\mathbf{I}^{\mathrm{b}} / \mathbf{E}^{\mathrm{c}}\right)\end{array}$ \\
\hline David P. van der Ham, $2012^{13}$ & Multicentro & $\mathrm{ECAC}^{\mathrm{d}}$ & Dic 2009 - Ene 2011 & $34-366 / 7$ & $95 / 100$ \\
\hline Jonathan M Morris, $2015^{14}$ & Multicentro & $\mathrm{ECAC}^{\mathrm{d}}$ & May 28, 2004, - Jun 2013 & $34-366 / 7$ & $923 / 912$ \\
\hline Susan M. Cox, $1995^{17}$ & EE.UU & $\mathrm{ECAC}^{\mathrm{d}}$ & May 1991 - Abr 1994 & $30-34$ & $61 / 68$ \\
\hline Jean Pasquier, $2009^{12}$ & NA & Cohorte prospectivo & Abr 1999 -Abr 2001 & $24-336 / 7$ & $133 / 170$ \\
\hline Jay D. Iams, $1985^{18}$ & EE.UU & $\mathrm{ECAC}^{\mathrm{d}}$ & Sep 1979 - Nov 1982 & $28-34$ & $38 / 35$ \\
\hline Brian M. Mercer, $1993^{16}$ & EE.UU & $\mathrm{ECAC}^{\mathrm{d}}$ & Mar 11991 - Jul 31, 1992 & $32-36$ & $46 / 47$ \\
\hline David P. van der Ham, $2012^{15}$ & Holanda & $\mathrm{ECAC}^{\mathrm{d}}$ & Ene 2007- 9 Sep 2009 & $34-366 / 7$ & $266 / 266$ \\
\hline Garite TJ, $1981^{19}$ & EE.UU & $\mathrm{ECAC}^{\mathrm{d}}$ & May 1977 - Jul 1980 & $28-34$ & $80 / 80$ \\
\hline Eroiz-Hernández J $1997^{11}$ & México & $\mathrm{ECAC}^{\mathrm{d}}$ & NA & $28-34$ & $30 / 28$ \\
\hline
\end{tabular}

$\left({ }^{a}\right)$ Ruptura prematura de membranas pretérmino. $\left({ }^{b}\right)$ Inducción del parto. $\left({ }^{c}\right)$ Manejo expectante. $\left({ }^{d}\right)$ Ensayo clínico aleatorizado controlado.

Resultados de cada estudio: La TABLA 2 muestra los riesgos relativos (RR) con su respectivo intervalo de confianza que presentó cada desenlace en los diferentes estudios incluidos en la revisión. Desenlaces primarios: Síndrome de distrés respiratorio: $\mathrm{La}$ incidencia de SDR fue también evaluada en los nueve estudios incluidos. En el estudio de Eroiz-Hernández $\mathrm{J}$, et al. ${ }^{11}$ se encontró un mayor riesgo asociado al manejo con IL ( $R R=2,01$; IC 95\%: 1,25-3,25), un resultado en el mismo sentido fue reportado por Morris J, et al. ${ }^{14}(\mathrm{RR}=1,25$; IC 95\%: 1,08-1,45). Sin embargo, opuesto a estos dos estudios, Cox S, et al ${ }^{17}$ encontraron una asociación protectora del IL $(\mathrm{RR}=$ 0,61; IC 95\%: 0,42-0,90), y los otros seis estudios no encontraron diferencias estadísticamente significativas en este evento entre ambos manejos. En el metanálisis, para esta exploración, el $\mathrm{I}^{2}$ fue de $60.5 \%$, siendo estadísticamente significativas la prueba ji-cuadrada de heterogeneidad y la prueba Tau-cuadrada $(\mathrm{p}<0.05)$. El estimador de asociación consolidado de IL versus EM no fue estadísticamente significativo $(R R=1,08$; IC 95\%: 0,89-1,31). FIGURA 2A. Sepsis neonatal: El riesgo de presentar NS fue evaluado en todos los nueve estudios revisados. En el estudio de Garite TJ, et al. ${ }^{19}$ se encontró un mayor riesgo de NS en las mujeres tratados con IL comparadas con las tratadas con EM $(\mathrm{RR}=1,85$; IC 95\%: 1,32-2,58). En contraste, en el estudio realizado por Mercer BM, et al. ${ }^{16}$ se encontró un menor riesgo asociado al manejo con IL comparado con $\mathrm{EM}(\mathrm{RR}=0,58$; IC 95\%: 0,38-0,89). Los demás estudios no encontraron diferencias estadísticamente significativas. En consecuencia, en el metanálisis no se encontraron diferencias estadísticamente significativas en la ocurrencia de este evento según el tratamiento administrado $(\mathrm{RR}=0,92$; IC 95\%: 0,61-1,39). Para esta asociación, el $\mathrm{I}^{2}$ fue de $77.6 \%$, 
siendo estadísticamente significativas la prueba jicuadrada de heterogeneidad y la prueba Tau- cuadrada $(p<0.05)$. Figura 2B. Desenlaces secundarios: Muerte neonatal: La mortalidad neonatal no fue la excepción en presentar variabilidad según cada estudio, y fue evaluada en ocho de los nueve trabajos incluidos. Solamente Cox S, et $\mathrm{al}^{17}(\mathrm{RR}=1,90$; IC 95\%: 1,25-2,88) y Eroiz-Hernández J, et al. ${ }^{11}(\mathrm{RR}=$ 2,06; IC 95\%: 1,40-3,01) hallaron un riesgo mayor y estadísticamente significativo de presentar muerte neonatal en pacientes con IL comparado con EM. Adicionalmente, en el estudio realizado por Van der Ham DP, et $\mathrm{al}^{15}$ no se presentaron muertes neonatales, $\mathrm{y}$ por eso fue excluido del metanálisis al no ser estimable su RR. El resto de estudios no encontraron diferencias estadísticamente significativas. En consecuencia, en el metanálisis no se encontraron tampoco diferencias estadísticamente significativas en la ocurrencia de muertes neonatales entre ambos eventos $(R R=1,34$;
IC 95\%: 0,93-1,93), en este caso el $\mathrm{I}^{2}$ fue de 67.0\%, siendo estadísticamente significativas la prueba ji-cuadrada de heterogeneidad y la prueba Taucuadrada $(p<0,05)$. FIGURA 2C. Corioamnionitis: La corioamnionitis fue evaluada en cinco de los nueve estudios revisados. Se encontró que el IL es un factor protector con un valor marginalmente significativo en el estudio de Van der Ham DP, et al. ${ }^{15}$ con un $(\mathrm{RR}=$ 0,78; IC 95\%: 0,61-1,00), en contraste del trabajo de Garite TJ, et al. ${ }^{19}$ donde el IL incremento el riesgo de corioamnionitis ( $R R=1,37$; IC 95\%: 1,02-1,86), en el resto de estudios no se encontraron diferencias estadísticamente significativas. Figura 2D. En consecuencia, el estimador obtenido en el metanálisis tampoco fue estadísticamente significativa $(R R=$ 0,88; IC 95\%:0,58-1,35). Para esta última asociación evaluada, $\mathrm{I}^{2}$ fue de $74.5 \%$, siendo estadísticamente significativas la prueba ji-cuadrada de heterogeneidad y la prueba Tau- cuadrada $(\mathrm{p}<0,05)$.

Tabla 2. Estimadores de efecto para cada desenlace analizado en cada estudio revisado.

\begin{tabular}{|c|c|c|c|c|c|c|c|c|c|c|c|c|}
\hline \multirow{2}{*}{ Estudio } & \multicolumn{3}{|c|}{ Síndrome de distrés respiratorio } & \multicolumn{3}{|c|}{ Sepsis neonatal } & \multicolumn{3}{|c|}{ Coriamnionitis } & \multicolumn{3}{|c|}{ Muerte neonatal } \\
\hline & $I^{a}(\%)$ & $E^{b}(\%)$ & (RR; IC95\%) & $\mathbf{I}^{\mathbf{a}}(\%)$ & $\mathbf{E}^{\mathrm{b}}(\%)$ & (RR; IC95\%) & $I^{a}(\%)$ & $\mathbf{E}^{\mathrm{b}}(\%)$ & (RR; IC95\%) & $I^{a}(\%)$ & $\mathbf{E}^{\mathrm{b}}(\%)$ & (RR; IC95\%) \\
\hline $\begin{array}{l}\text { David P. van } \\
\text { der Ham }{ }^{13}\end{array}$ & $6(6)$ & $5(5)$ & $(1,13 ; 0,64-1,97)$ & $1(1)$ & $2(2,0)$ & $(0,68 ; 0,14-3,4)$ & NA & NA & NA & $1(1)$ & 0.0 & $(1,55 ; 0,69-3,49)$ \\
\hline $\begin{array}{l}\text { Jonathan M } \\
\text { Morris }^{14}\end{array}$ & $76(8)$ & $47(5)$ & $(1,25 ; 1,08-1,45)$ & $23(2)$ & $29(3)$ & $(0,88 ; 0,64-1,19)$ & NA & NA & NA & $73(8)$ & $61(7)$ & $\begin{array}{c}(1,09 ; 0,93- \\
1,28)\end{array}$ \\
\hline $\begin{array}{l}\text { Susan } \\
\text { M.Cox }{ }^{17}\end{array}$ & $23(37)$ & $41(59)$ & $(0.61 ; 0,42-0,90)$ & $2(3)$ & $5(7)$ & $(0,59 ; 0,18-1,93)$ & $1(2)$ & $10(15)$ & $(0,18 ; 0,3-1,17)$ & $3(5)$ & $0(0)$ & $(1,90 ; 1,25-2,88)$ \\
\hline $\begin{array}{l}\text { Jean } \\
\text { Pasquier }^{12}\end{array}$ & $36(27,1)$ & $40(23,5)$ & $(1,11 ; 0.84-1,47)$ & $2(1,5)$ & $10(5,9)$ & $(0,37 ; 0,10-1,32)$ & NA & NA & NA & $2(1,5)$ & $5(2,9)$ & $(0,65 ; 0,20-2,10)$ \\
\hline Jay D. Iams ${ }^{18}$ & $10(26,3)$ & $12(34,3)$ & $(0,83 ; 0,49-1,39)$ & $4(10,5)$ & $3(8,6)$ & $(1,11 ; 0,56-2,20)$ & NA & NA & NA & $1(2,6)$ & $1(2,9)$ & $(0,96 ; 0,24-3,91)$ \\
\hline Brian Mercer ${ }^{16}$ & $2(4,4)$ & $1(2,1)$ & $(1,36 ; 0,60-3,12)$ & $18(39)$ & $31(65)$ & $(0,58 ; 0,38-0,89)$ & $5(10,9)$ & $13(27,7)$ & $(0,51 ; 0,23-1,10)$ & NA & NA & NA \\
\hline $\begin{array}{l}\text { David P. van } \\
\text { der Ham }{ }^{15}\end{array}$ & $21(7,8)$ & $17(6,3)$ & $(1,11 ; 0,83-1,50)$ & $7(2,6)$ & $11(4,1)$ & $(0,77 ; 0,43-1,39)$ & $43(22)$ & $62(32)$ & $(0,78 ; 0,61-1,0)$ & $0(0)$ & $0(0)$ & NA \\
\hline Garite $\mathrm{TJ}^{19}$ & $14(17,5)$ & $17(21,3)$ & $(0,88 ; 0,58-1,35)$ & $4(5)$ & $0(0)$ & $(2,05 ; 1,75-2,41)$ & $34(42,5)$ & $22(27,5)$ & $(1,37 ; 1,02-1,86)$ & $2(2,5)$ & $4(5)$ & $(0,66 ; 0,21-2,06)$ \\
\hline $\begin{array}{l}\text { Eroiz- } \\
\text { Hernández } \mathbf{J}^{11}\end{array}$ & $16(53)$ & $5(18)$ & $(2,01 ; 1,25-3,25)$ & $2(7)$ & $0(0)$ & $(2,00 ; 1,54-2,60)$ & $10(33)$ & $7(25)$ & $(1,21 ; 0,73-2,00)$ & $9(30)$ & $1(4)$ & $(2,06 ; 1,40-3,01)$ \\
\hline
\end{tabular}

( ${ }^{\mathrm{a}}$ Inducción de parto. $\left({ }^{\mathrm{b}}\right)$ Manejo expectante.

La Tabla 3, presenta la meta-regresión para cada desenlance, usando como predictora la edad gestacional categorizada $(1=28-34$ semanas, $0=$ mayor de 34$)$. No se observó impacto de la variable edad gestacional sobre el estimador meta-analítico obtenido para ninguno de los desenlances estudiados. Finalmente, en la Figura 3, se presenta los Funnel-plot del test de Egger, observándose una simetría en la distribución de los estimadores que no es compatible con el sesgo de publicación, aunque el bajo número de estudios para los últimos dos descenlaces dificulta su interpretación. 
Inducción del parto versus manejo expectante en ruptura prematura de membranas pretérmino: revisión sistemática y metanálisis
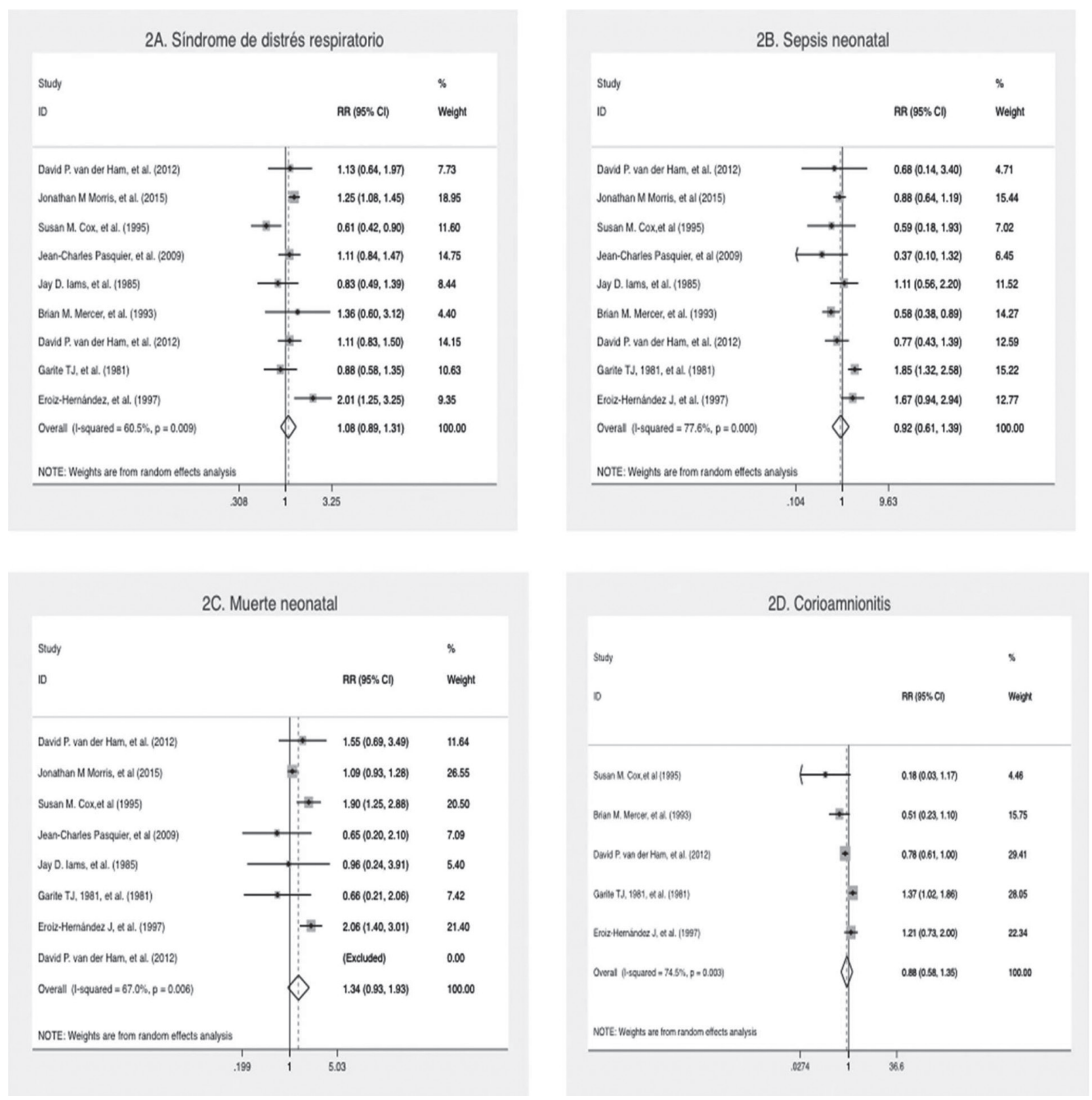

Figuras 2. Metanálisis por evento.

Tabla 3. Estimadores de obtenidos de la meta-regresión de cada desenlace usando como variable independiente principal (1= 28-34 semanas, $0=$ mayor de 34).

\begin{tabular}{lcc}
\hline Evento & Beta (IC 95\%) & Valor de p \\
\hline Síndrome de distres respiratorio & $0.74(0.38-1.45)$ & 0.37 \\
Sepsis neonatal & $1.42(0.72-2.82)$ & 0.30 \\
Muerte neonatal & $2.18(0.18-25.79)$ & 0.52 \\
Corioamnionitis & $2.05(0.80-5.21)$ & 0.12 \\
\hline
\end{tabular}



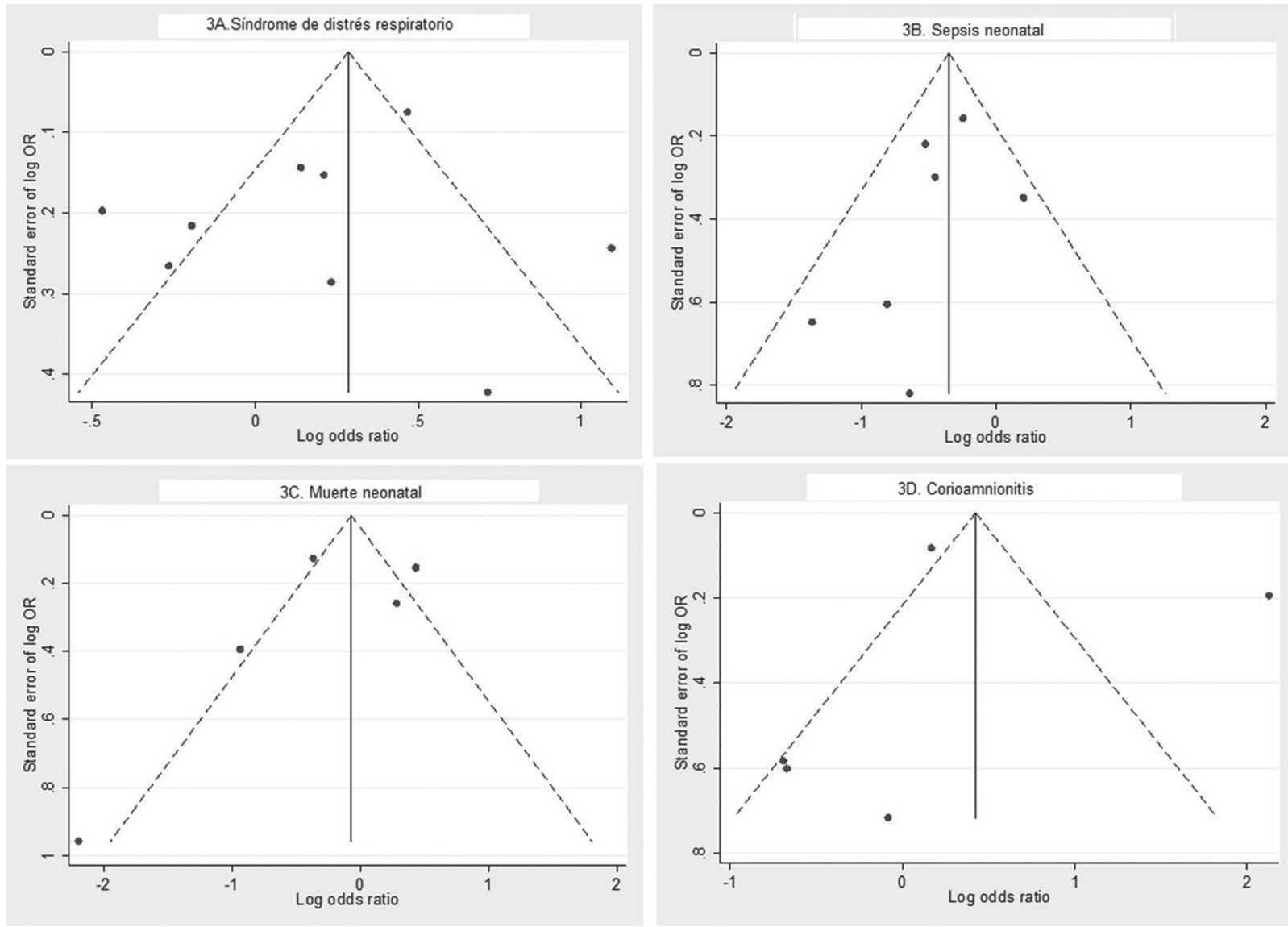

Figura 3. Egger test para cada desenlace analizado.

\section{DISCUSIÓN}

Los resultados encontrados en esta revisión y metaanálisis, para lograr determinar el manejo óptimo para las pacientes complicadas con PPROM siguen siendo controversiales. Los resultados sugieren que no existe evidencia de diferencias estadísticamente significativas en la ocurrencia de los principales desenlaces cuando se compara EM con IL. Esto se debe a los diferentes hallazgos de los estudios analizados, los cuales están directamente relacionados, en primer lugar con las divergencias entre ellos; ya sea por la semana gestacional donde se presentó la PPROM, como por el uso de antibióticos profilácticos, el uso de esteroides para maduración pulmonar, el llevar a término las pacientes con manejo expectante, las horas de latencia desde la ruptura de membranas hasta el parto, el uso de tocolíticos u oxitocina. Es decir, parte de la alta heterogeneidad encontrada en esta revisión, se explica por las diferencias metodológicas entre los estudios, así como porque las intervenciones no se aplican estrictamente en las mismas condiciones, pero dado el bajo tamaño de muestra, y la no escasez de

información en la mayoría de estudios, estas fuentes de heterogeneidad no pudieron ser evaluadas en esta metanálisis, exceptuando la edad gestacional, en la cuál en todo caso, no se encontró un impacto sobre los estimadores encontrados.

Entre los hallazgos de esta revisión, se observó que las pacientes tratadas con IL no presentaron diferencias estadísticamente significativamente en la ocurrencia de SDR comparado con aquellas pacientes que recibieron EM. Si observamos estudio por estudio en la mayoría de estos se encontró un riesgo de presentar SDR, sin embargo no en todos ellos fue estadísticamente significativo y a su vez, otros estudios encontraron resultados divergentes. No obstante, el manejo complementario dado y las circunstancias propias de la población pudieron haber condicionado los diferencias de los resultados. Por ejemplo, en el ensayo clínico desarrollado por Morris J, et al. ${ }^{14}$, las pacientes con EM fueron llevadas a término cumpliendo así con la maduración pulmonar mientras que las gestantes con IL no recibieron esteroides antenatales. Un caso similar, es el de Eroiz-Hernández J, et al. ${ }^{18}$, quienes realizaron 
maduración pulmonar, pero las pacientes incluidas en el estudio presentaban PPROM temprana (semanas 2834). Esta falta de asociación estadística también fue encontrada en un metanálisis realizado por Buchanan $\mathrm{S}$, et al. ${ }^{20}$, donde el riesgo fue de $(\mathrm{RR}=0,98$; IC 95\%: $0,74-1,29)$ y en otro realizado por Hazem Al-Mandeel, et $\mathrm{al}^{21}$ con un $(\mathrm{RR}=1,13$; IC $950,78-1,63)$ para el grupo tratado con IL frente al EM.

Al igual que con el SDR se evidenció que la IL no presentaba diferencia estadísticamente significativas con respecto a la ocurrencia de NS frente a las pacientes a quienes se les fue realizado un EM. Llama la atención los resultados obtenidos por Mercer BM, et al. ${ }^{16}$, los cuales asocian la IL con una reducción en el riesgo para la aparición de NS, sin embargo, hay que tener en cuenta que en el desarrollo de este estudio no se realizó profilaxis antibiótica y, una de las indicaciones para el parto en las pacientes con EM fue la presencia de corioamnionitis, razón por la cual se pudo haber aumentado la relación de NS con EM. Los metanálisis de Buchanan S, et al. ${ }^{20}$ y Al-Mandeel $\mathrm{H}$, et al. ${ }^{21}$ tampoco obtuvieron asociación con significancia estadística para el desenlace de sepsis neonatal al compara la IL y el manejo expectante con un $(\mathrm{RR}=1,33$; IC 95\%: 0,72$2,47)$ y $(R R=1,49$; IC 95\%: 0.57-3.88) respectivamente.

Por lo que se refiere a corioamnionitis, no se encontró una asociación estadísticamente significativa en las pacientes a quienes se les realizó IL en comparación con aquellas pacientes que fueron tratadas con un EM, cabe resaltar que para este desenlace el estudio realizado por Garite TJ, et al. ${ }^{19}$ encontró un riesgo aumentado en las pacientes manejadas con IL, esto puede ser el resultado de una larga latencia desde la PPROM hasta el momento del parto de éste grupo, y a la no administración de antibióticos de manera profiláctica. Respecto a corioamnionitis Al-Mandeel $\mathrm{H}$, et $\mathrm{al}^{21}$ realizaron dos estimaciones una con todos los estudios incluidos en la revisión y otro solo con los estudios que no usaron esteroides antenatales y en ninguna de las dos encontraron asociación estadística $(\mathrm{RR}=1,22$; IC 95\%: 0,69-2,25 y (RR=0.83; IC 95\%: 0,35-1,95). Buchanan $\mathrm{S}$, et al. ${ }^{20}$, de igual manera que en nuestro estudio ellos no evidencian que la IL se encuentre asociada con corioamnionitis ( $R R=0,44$; IC 95\%: 0,17-1,14) al comparar con el EM. Ellos realizaron un análisis de subgrupos y compararon el IL temprano (menor a 24h) y el tardío (mayor a 24h) revelando una asociación estadísticamente significativa mostrando como factor protector a la IL temprana ( $R R=0,25$; IC del 95\%: 0,10$0,61)$, lo que sugiere que entre más corto sea el tiempo de latencia entre la PPROM y el parto menor va a ser la incidencia de infección materna.

Por último, la mortalidad fetal tampoco presentó asociación estadísticamente significativa al comparar ambos grupos de tratamiento. Cox S, et al. ${ }^{17}$ y EroizHernández $\mathrm{J}$, et al. ${ }^{11}$ asociaron de manera significativa la IL con un aumento del riesgo de presentar muerte neonatal, estos dos estudios poseen similitudes en cuanto a la edad gestacional en la cual se presento la PPROM de las pacientes incluidas, la cual es menor a 34 semanas y que en ambos estudios no se usaron esteroides antenatales, lo que nos sugiere que el uso de esteroides antenatales en las pacientes tratadas con PPROM antes de la semana 34 puede disminuir el riesgo de presentar muerte neonatal. El hallazgo de Al-Mandeel $\mathrm{H}$, et $\mathrm{al}^{21}$ en su metanálisis, al igual que en nuestra revisión no encontraron un relación significativa entre la muerte neonatal y la IL, adicionalmente ellos realizaron otro metanálisis donde solo incluyeron los estudios que no usaron esteroides antenatales encontrando una asociación estadísticamente significativa entre IL y la muerte neonatal comparada con el $\mathrm{EM}(\mathrm{RR}=5,81$; IC 95\%: 1,35-25,08).

El único estudio de cohorte incluido, fue el de Pasquier $\mathrm{J}$, et al. ${ }^{12}$, destacando que el grupo IL incluyó pacientes con más de 24 horas post-ruptura de membranas, al igual que el estudio Iams JD, et al. ${ }^{18}$, Garite TJ, et al. ${ }^{19} \mathrm{y}$ Eroiz-Hernandez J, et al. ${ }^{11}$, mientras que en el resto de estudios la inducción se realizó dentro de las primeras 24 horas, posterior a la aleatorización. Por otra parte, en el estudio de cohortes la muestra estaba compuesta de pacientes que cursaban PPROM con una edad gestacional entre 24 y 34 semanas de edad gestacional, resaltando que fue el estudio con inclusión de pacientes con ruptura más temprana y por ende, menor desarrollo fetal y más complicaciones para el neonato debido a la prematuridad extrema. Otra de las diferencias de este trabajo con respecto a la totalidad de los estudios, fue la en el tamaño de la muestra entre el grupo de intervención y el de comparación.

De todos los artículos, cinco tuvieron una asociación estadísticamente significativa para la inducción del parto, como factor de riesgo o factor protector en alguno de los cuatro desenlaces a excepción de los estudios de Iams JD, et al. ${ }^{18}$, Pasquier J, et al. ${ }^{12}$ y ambos estudios de Van der Ham DP, et al. ${ }^{13,15}$. En el caso de Iams JD, et al. ${ }^{18}$, esto puede ser debido a que en el grupo de IL se emplearon esteroides antenatales además de tocolíticos hasta completar el esquema de maduración $\mathrm{y}$, se dio terapia antibiótica anteparto a las pacientes con signos de infección materna (corioamnionitis 
clínica, urocultivo positivo); De igual forma, en el estudio de Pasquier J, et $\mathrm{al}^{12}$, esto puede verse explicado por el hecho de ser un estudio de cohortes, y por lo tanto, dificultad en el establecimiento de un manejo determinado y comparable para cada grupo de intervención, o a confusión. Por último, en cuanto a los estudios de Van der Hamm DP, et al. ${ }^{13,15}$, ambos poseen una metodología muy similar, tomando como muestra pacientes con PPROM tardía además del uso de medidas adicionales como terapia antibiótica ante signos de infección y monitoreo continuo, consideraciones que pudieran llevar a disminuir alguno de los desenlaces a evaluar y, por ello no encontrar una asociación significativa.

Cabe mencionar además que en países en desarrollo como Colombia, la costo-efectividad que implica el manejo de la PPROM es un aspecto importante a tener en cuenta. Vijgen SM, et al..$^{22}$ elaboraron un análisis secundario de un ECAC, encontrando que las pacientes tratadas con IL tuvieron un tratamiento más costoso comparado con EM. Esta diferencia se dio principalmente en los costos del parto y del periodo post-parto. A su vez, los costos del manejo anteparto fueron más altos en las pacientes tratadas con EM.

Fortalezas y limitaciones: Las limitaciones de nuestro estudio se basan principalmente en los escasos estudios existentes para realizar la revisión. Este problema también lo presentaron Buchanan $\mathrm{S}$, et al..$^{20} \mathrm{y}$ $\mathrm{Al}-$ Mandeel $\mathrm{H}$, et al. ${ }^{21}$, añadiendo a sus revisiones siete y cinco estudios respectivamente, nosotros incluimos nueve, haciendo la salvedad que uno de ellos fue un estudio de cohortes. Otro problema identificado fue la diversidad del manejo dado a cada uno de los grupos de comparación en los artículos revisados puesto que variaba en cuanto a los cuidados adicionales, generando así disparidad en los hallazgos. Otra limitación tiene que ver con el bajo tamaño de la muestra incluida, y el hecho de que la mayoría de eventos son raros. Adicionalmente la no existencia de información en la mayoría de trabajos sobre otras covariables relevantes como uso de antibióticos, uso de corticoides u horas de latencia entre la PPROM y el parto, impidieron estudiar la modificación de la asociación por estas covariables; y así mismo esto pudo afectar el poder estadístico de las meta-regresiones realizadas para la edad gestacional. Con respecto a las fortalezas, nuestro artículo se desarrolló sin limitación de lenguaje ni espacio temporal; la selección de artículos de forma pareada con un tercer examinador final; y el proceso de redacción se hizo bajo los lineamientos de la escala PRISMA, cumpliendo con 23 de los 27 ítems propuestos.

\section{CONCLUSIONES}

En los estudios analizados no se encontraron diferencias significativas en cuanto al SDR, NS, muerte neonatal y corioamnionitis según el manejo realizado (IL o EM) a pacientes embarazadas complicadas con PPROM. No obstante, los escasos de los estudios disponibles para la revisión no son suficientes y su variabilidad en la metodología impide que se puedan realizar recomendaciones para guiar el tratamiento de paciente complicadas con PPROM. Por lo mencionado anteriormente, se hace necesario la realización de ECAC con uniformidad en los procedimientos realizados a cada grupo de exposición para de esta manera lograr que los resultados obtenidos puedan ser comparables entre estudio y estudio, y de esta manera poder corroborar los resultados encontrados en esta revisión y despejar las dudas que no pudieron ser resueltas.

\section{AGRADECIMIENTOS}

Le agradecemos a la Universidad Industrial de Santander por propiciar espacios de investigación que nos motivaron a realizar esta revisión.

\section{CONFLICTO DE INTERESES}

Los autores no reportan conflicto de intereses.

\section{REFERENCIAS}

1. Mercer BM. Preterm premature rupture of the membranes: current approaches to evaluation and management. Obstet Gynecol Clin North Am. 2005; 32(3): 411. DOI: 10.1016/j.ogc.2005.03.003.

2. Dars S, Malik S, Samreen I, Kazi R. Maternal morbidity and perinatal outcome in preterm premature rupture of membranes before 37 weeks gestation. Pak J Med Sci. 2014; 30(3): 626-629. DOI: $10.12669 /$ pjms.303.4853.

3. Joy S, Nair S, Radhamany K. Impact of fetal presentation on pregnancy outcome in preterm premature rupture of membranes. J Clin Diagn Res. 2014; 8(11): OC03-OC06. DOI: 10.7860/ JCDR/2014/9553.5114.

4. Beck S, Wojdyla D, Say L, Betran AP, Merialdi $\mathrm{M}$, Requejo $\mathrm{JH}$, et al. The Worldwide incidence of preterm birth: a systematic review of maternal mortality and morbidity. Bull World Health Organ. 2010; 88:31-38. DOI: 10.2471/BLT.08.062554.

5. Kacerovsky M, Musilova I, Andrys C, Hornychova $\mathrm{H}$, Pliskova L, Kostal M, et al. Prelabor rupture of membranes between 34 and 37 weeks: the 
intraamniotic inflammatory response and neonatal outcomes. Am J Obstet Gynecol. 2014; 210(4): 325. e1-325.e10. DOI: 10.1016/j.ajog.2013.10.882.

6. Gezer A, Parafit-Yalciner E, Guralp O, Yedigoz V, Altinok T, Madazli R. Neonatal morbidity mortality outcomes in pre-term premature rupture of membranes. J Obstet Gynaecol. 2013; 33(1): 38-42. DOI: $10.3109 / 01443615.2012 .729620$.

7. DANE: Departamento Administrativo Nacional de Estadística. Bogotá. DANE; Defunciones fetales por tiempo de gestación, según departamento de residencia de la madre y grupos de causas de defunción.

8. Simhan HN, Canavan TP. Preterm premature rupture of membranes: diagnosis, evaluation and management strategies. BJOG.2005; 112(1): 32-37. DOI: $10.1111 / \mathrm{j} .1471-0528.2005 .00582 . x$.

9. Schutte MF, Treffers PE, Kloosterman GJ, Soepatmi S. Management of premature rupture of membranes: the risk of vaginal examination to the infant. Am J Obstet Gynecol. 1983; 146(4): 395-400.

10. Mateus J, Fox K, Jain S, Jain S, Latta R, Cohen J. Preterm Premature Rupture of Membranes: Clinical Outcomes of Late-Preterm Infants. Clin Pediatr (Phila). 2010; 49(1): 60-65. DOI: 10.1177/0009922809342460.

11. Eroiz-Hernandez J, Trejo-Acuña MA, AlvarezTarín MH. [Conservative management of premature ruptura in pregnancy of 28-34 weeks. Aleatory clinical trial]. Ginecol Obstet Mex 1997; 65: 43-47.

12. Pasquier J, Picaud J. Neonatal outcomes after elective delivery management of preterm premature rupture of the membranes before 34 weeks' gestation (DOMINOS study). Eur J Obstet Gynecol Reprod Biol. 2009; 143(1): 18-23. DOI: 10.1016/j. ejogrb.2008.10.017.

13. Van der Ham D, Van der Heyden J, Opmeer B. Management of late-preterm premature rupture of membranes: the PPROMEXIL-2 trial. Am J Obstet Gynecol. 2012; 207(4): 276.e1-276.e10. DOI: 10.1016/j.ajog.2012.07.024.

14. Morris J, Roberts C, Bowen J. Immediate delivery compared with expectant management after preterm pre-labour rupture of the membranes close to term (PPROMT trial): a randomised controlled trial. Lancet. 2016; 387(10017): 444-452. DOI: 10.1016/ S0140-6736(15)00724-2.

15. van der Ham DP, Vijgen SM, Nijhuis JG, van Beek JJ, Opmeer BC, Mulder AL, et al. Induction of labor versus expectant management in women with preterm prelabor rupture of membranes between 34 a 37 weeks: a randomized controlled trial. PLoS
Med. 2012; 9(4): e1001208. DOI: 10.1371/journal. pmed.1001208.

16. Mercer BM, Crocker LG, Boe NM, Sibai BM. Induction versus expectant management in premature rupture of the membranes with mature amniotic fluid at 32 to 36 weeks: a randomized trial. Am J Obstet Gynecol. 1993; 169(4): 775-782.

17. Cox S, Leveno K. Intentional delivery versus expectant management with preterm ruptured membranes at $30-34$ weeks' gestation. Obstet Gynecol. 1995; 86(6): 875-879. DOI: 10.1016/00297844(95)00303-9.

18. Iams JD, Talbert ML, Barrows H, Sachs L. Management of preterm prematurely ruptured membranes: A prospective randomized comparison of observation versus use of steroids and timed delivery. Am J Obstet Gynecol. 1985; 151(1): 32-38.

19. Garite TJ, Freeman RK, Linzey EM, Braly PS, Dorchester WL. Prospective randomized study of corticosteroids in the management of premature rupture of the membranes and the premature gestation. Am J Obstet Gynecol. 1981; 141(5): 508-515.

20. Buchanan S, Crowther C, Levett K, Middleton $\mathrm{P}$, Morris J. Planned early birth versus expectant management for women with preterm prelabour rupture of membranes prior to 37 weeks' gestation for improving pregnancy outcome. Cochrane Database Syst Rev. 2010; (3): CD004735. DOI: 10.1002/14651858.CD004735.pub3.

21. Al-Mandeel H, Alhindi MY, Sauve R. Effects of intentional delivery on maternal and neonatal outcomes in pregnancies with preterm prelabour rupture of membranes between 28 and 34 weeks of gestation: a systematic review and meta-analysis. J Matern Fetal Neonatal Med. 2013; 26(1): 83-89. DOI: $10.3109 / 14767058.2012 .718388$.

22. Vijgen SM, van der Ham DP, Bijlenga D, van Beek JJ, Bloemenkamp KW, Kwee A, et al. Economic analysis comparing induction of labor and expectant management in women with preterm prelabor rupture of membranes between 34 and 37 weeks (PPROMEXIL trial). Acta Obstet Gynecol Scand. 2014; 93(4): 374-381. DOI: 10.1111/aogs.12329. 